\title{
A survey of analgesic and anti-inflammatory drug prescription for oral implant surgery
}

\author{
Rahul Datta ${ }^{1}$, Yasmin Grewal ${ }^{2}$, Jaspreet Singh Batth ${ }^{3}$, Amandeep Singh ${ }^{4}$ \\ ${ }^{1}$ Department of Oral and Maxillofacial Surgery, Rayat Bahra Dental College and Hospital, Mohali 140104, Punjab, India. \\ ${ }^{2}$ Department of Public Health Dentistry, Rayat Bahra Dental College and Hospital, Mohali 140104, Punjab, India. \\ ${ }^{3}$ Department of Conservative Dentistry and Endodontics, BRS Dental College and Hospital, Panchkula 134109, Punjab, India. \\ ${ }^{4}$ Department of Pharmacology, Shri Guru Ram Rai Institute of Medical and Health Sciences, Patel Nagar, Dehradun 248001, Uttarakhand, India.
}

Address for correspondence: Dr. Rahul Datta, Department of Oral and Maxillofacial Surgery, Rayat Bahra Dental College and Hospital, Mohali 140104, Punjab, India. E-mail: docdatta@gmail.com

\begin{abstract}
Aim: This study was conducted to determine the preferred analgesic and anti-inflammatory drugs prescribed by oral implantologists in India. Methods: A structured questionnaire was distributed to 332 dentists to gather information regarding their prescription habits for analgesics and anti-inflammatory drugs. Frequency distributions were computed by type of drug being prescribed and the protocol followed. Results: Analysis of data showed that majority of dentists $(85.8 \%, n=285)$ prescribed conventional non-steroidal anti-inflammatory drugs (NSAIDs) for implant surgery. The most common prescription was ibuprofen with paracetamol combination $(32.2 \%, n=107)$ followed by diclofenac $(20.2 \%, n=67)$. Most dentists reported prescribing different NSAIDs for the same procedure in different patients $(64.7 \%, n=215)$. Only, $35.5 \%(n=118)$ followed the peri-operative protocol. Adjunctive prescription of steroids was done by only $33.7 \%(n=112)$. Conclusion: Our study illustrates that the general trend of analgesic and anti-inflammatory drug prescription for dental implant surgery among Indian dentists is mostly in accordance with the guidelines for pain management worldwide. However, it is noteworthy that a few dentists do prescribe drugs not primarily indicated for dental pain management and use widely varying protocols for the same. Therefore, in order to avoid potential complications, it is essential to raise awareness of among the dental practitioners of the appropriate indications and dosage regimen of specific drugs.
\end{abstract}

Key words:

Dental implants, non-steroidal anti-inflammatory drugs prescription, steroid, protocol

\section{INTRODUCTION}

Pain management has always been an important part of dental care. With an estimated 30 million people benefited worldwide, non-steroidal anti-inflammatory drugs (NSAIDs) are the most commonly used drugs for managing surgical pain and inflammation. Consistently, NSAIDs are routinely prescribed for mild to moderate dental pain and will be supplemented/replaced by opioid analgesics in severe

\begin{tabular}{|l|l|}
\multicolumn{2}{|c|}{ Access this article online } \\
\hline Quick Response Code: & Website: \\
\hline & www.parjournal.net \\
\cline { 2 - 3 } & \\
\hline
\end{tabular}

pain. The role of steroids as adjunctive measures to reduce postoperative inflammation, swelling, and pain has also received importance in recent years. ${ }^{[1-4]}$

Despite adherence to all surgical recommendations and precautions during dental implant surgery in a normal healthy patient, many patients experience mild to moderate pain and inflammation after the procedure. ${ }^{[5,6]}$ Though the role of NSAIDs and steroids in minimizing postoperative discomfort in dentoalveolar surgery has been reviewed extensively, specific recommendations regarding drugs, doses, and protocols with respect to dental implant surgery are not defined. ${ }^{[7,8]}$

A comprehensive search of commonly used electronic databases such as PubMed and Google Scholar, using the key words "analgesics", "anti-inflammatory" and "oral implants" was done. Published literature in this context related either to the use of analgesic or anti-inflammatory 
drugs by dental practitioners in routine clinical practice, with no specific relation to oral implant surgery.

Although the role of NSAIDs and steroids has been very beneficial in terms of pain relief, these drugs also have an associated risk of side effects and adverse drug reactions. ${ }^{[5]}$ Since safe usage of drugs in clinical practice entails maximizing the therapeutic efficacy and minimizing the adverse effects, it is important to gain knowledge of the pattern of use of these widely prescribed drugs by dentists in order to minimize the possible risks associated with these drugs. ${ }^{[1-4]}$ The aim of our study was therefore to assess the prescription pattern of analgesics and anti-inflammatory drugs during routine oral implant surgery in normal healthy patients among Indian dentists. To our knowledge, this is the first study of its kind to be conducted in the Indian subcontinent.

\section{METHODS}

A structured questionnaire was developed to elicit prescription preferences regarding analgesics and anti-inflammatory drugs prescribed for routine oral implant surgery in the normal healthy patients and the protocol(s) followed. Dental surgeons were personally approached at various national events such as conferences and academic meetings and requested to provide the required information. Inclusion criteria for the study sample consisted of dental surgeons performing oral implant surgery and were willing to voluntarily complete the questionnaire. All data was manually transferred from the survey forms to an electronic spreadsheet for further analysis.

\section{RESULTS}

Out of a total of 332 dentists that responded to the survey, all study participants stated that they prescribed analgesic and anti-inflammatory drugs for implant surgery to their patients. Nine NSAIDs, acetaminophen (paracetamol) and two semi-synthetic opioid drugs were prescribed [Table 1]. Additionally, 33.7\% $(n=112)$ participants prescribed steroids in conjunction with NSAIDs [Table 2].

The most preferred prescription was the combination of ibuprofen and paracetamol $(32.2 \%, n=107)$ [Figure 1]. The most commonly prescribed drugs were ibuprofen $(44.3 \%$, $n=147)$ and diclofenac $(33.7 \%, n=112)$. Paracetamol was prescribed by $54.2 \%(n=180)$ of the dentists, however, all of these dentists prescribed paracetamol

Table 1: Profile of analgesic and anti-inflammatory drug prescription by dentists performing oral implant surgery

\begin{tabular}{|c|c|c|c|c|c|c|c|c|c|c|c|c|}
\hline \multirow[t]{2}{*}{ Drug name } & \multirow[t]{2}{*}{$n$} & \multicolumn{3}{|c|}{ Drug name used } & \multicolumn{3}{|c|}{ Protocol } & \multicolumn{3}{|c|}{ Route } & \multirow{2}{*}{$\begin{array}{l}\text { Additional } \\
\text { steroid } \\
\text { prescription }\end{array}$} & \multirow{2}{*}{$\begin{array}{c}\text { Unrelated } \\
\text { drugs } \\
\text { prescribed }\end{array}$} \\
\hline & & Generic & Trade name & Both & Peri & Post & sos & Oral & IM & IV & & \\
\hline Ibuprofen+PCM & 107 & 34 & 66 & 7 & 37 & 70 & 23 & 107 & 0 & 0 & 19 & 29 \\
\hline Diclofenac & 67 & 50 & 13 & 4 & 23 & 44 & 16 & 67 & 7 & 3 & 26 & 15 \\
\hline Diclofenac+PCM & 45 & 26 & 13 & 6 & 23 & 22 & 9 & 45 & 0 & 0 & 7 & 24 \\
\hline Ibuprofen & 40 & 31 & 8 & 1 & 18 & 22 & 9 & 40 & 0 & 0 & 13 & 9 \\
\hline NSAIDs (unspecified) & 28 & 28 & 0 & 0 & 5 & 23 & 9 & 28 & 2 & 0 & 6 & 0 \\
\hline Ketorolac & 22 & 1 & 21 & 0 & 4 & 18 & 4 & 22 & 1 & 0 & 7 & 8 \\
\hline Piroxicam & 18 & 11 & 4 & 3 & 8 & 10 & 2 & 18 & 0 & 0 & 5 & 6 \\
\hline Tramadol & 14 & 9 & 1 & 4 & 3 & 11 & 2 & 14 & 2 & 4 & 9 & 12 \\
\hline Aceclofenac+PCM & 14 & 8 & 4 & 2 & 7 & 7 & 2 & 14 & 0 & 0 & 8 & 8 \\
\hline Nimesulide+PCM & 12 & 3 & 9 & 0 & 6 & 6 & 6 & 12 & 0 & 0 & 2 & 4 \\
\hline Nimesulide & 10 & 7 & 3 & 0 & 1 & 9 & 0 & 10 & 0 & 0 & 3 & 3 \\
\hline Aceclofenac & 9 & 9 & 0 & 0 & 1 & 8 & 4 & 9 & 0 & 0 & 4 & 9 \\
\hline Dextropropoxyphene & 5 & 0 & 5 & 0 & 2 & 3 & 2 & 5 & 0 & 0 & 0 & 0 \\
\hline Tramadol+PCM & 2 & 1 & 1 & 0 & 2 & 0 & 0 & 2 & 0 & 0 & 2 & 0 \\
\hline Etoricoxib & 2 & 2 & 0 & 0 & 2 & 0 & 0 & 2 & 0 & 0 & 0 & 2 \\
\hline Aspirin & 2 & 2 & 0 & 0 & 0 & 2 & 0 & 2 & 0 & 0 & 0 & 0 \\
\hline Mefenamicacid+PCM & 1 & 0 & 1 & 0 & 0 & 1 & 0 & 1 & 0 & 0 & 1 & 1 \\
\hline Total & & 222 & 144 & 27 & 18 & 140 & 253 & 86 & 393 & 12 & 7 & 112 \\
\hline
\end{tabular}

PCM: Paracetamol, Peri: Peri-operative, Post: Post-operative route, PO: Per oral, IV: Intra venous, IM: Intra muscular, NSAIDs: Non-steroidal anti-inflammatory drugs, SOS: Stat on symptoms

Table 2: Profile of steroid drug prescription by dentists performing oral implant surgery

\begin{tabular}{|c|c|c|c|c|c|c|c|c|c|c|}
\hline \multirow[t]{2}{*}{ Drug name } & \multirow[t]{2}{*}{$n$} & \multicolumn{3}{|c|}{ Drug name used } & \multicolumn{3}{|c|}{ Protocol } & \multicolumn{3}{|c|}{ Route } \\
\hline & & Generic & Trade name & Both & Pre & Peri & Post & Oral & IM & IV \\
\hline Prednisolone & 33 & 28 & 5 & 0 & 3 & 7 & 23 & 107 & 0 & 0 \\
\hline Dexamethasone & 57 & 42 & 15 & 0 & 19 & 2 & 36 & 67 & 7 & 3 \\
\hline Hydrocortisone & 2 & 2 & 0 & 0 & 2 & 0 & 0 & 45 & 0 & 0 \\
\hline Betamethasone & 20 & 9 & 11 & 0 & 10 & 3 & 7 & 40 & 0 & 0 \\
\hline Total & 112 & 81 & 31 & 0 & 34 & 12 & 66 & 28 & 2 & 0 \\
\hline
\end{tabular}

Pre: Pre-operative, Peri: Peri-operative, Post: Post-operative, Route: PO: Per oral, IV: Intra venous, IM: Intra muscular 


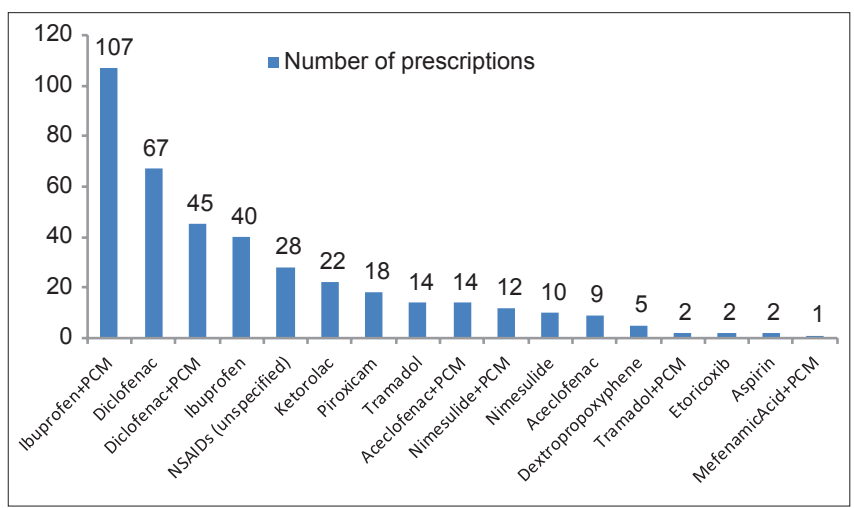

Figure 1: Prescription trend of analgesic/anti-inflammatory drugs for oral implant surgery. PCM: Paracetamol

as an additional drug in combination with some other analgesic, and in no case was paracetamol used as an analgesic-anti-inflammatory drug alone.

More than half of the dentists prescribed more than one drug $(64.7 \%, n=215)$, and of these $8.4 \%(n=28)$ simply stated that they prescribed NSAIDs, without specifying any drug or trade name. Also, majority $(56.9 \%, n=189)$ used generic drugs names in their prescriptions, brand name was used by $37.3 \%(n=124)$ and $5.7 \%$ mentioned both $(n=19)$. A single NSAID drug was prescribed by only $35.2 \%(n=117)$ dentists. Unrelated drugs were mentioned by $27.4 \%(n=91)$ dentists and $58.1 \%(n=193)$ indicated the use of pre-formulated drug combinations.

The use of semi-synthetic opioids was noted by 9.0\% $(n=30)$ dentists. Tramadol alone was prescribed by $3.3 \%(n=11)$ dentists while $1.5 \%(n=5)$ gave it in combination with paracetamol. Dextropropoxyphene was prescribed by $1.5 \%(n=5)$ dentists only and it was part of a pre-formulated commercially available drug formulation.

Additional drugs were also prescribed along with analgesic-anti-inflammatory drugs. These included tissue enzymes such as serratiopeptidase $(10.8 \%, n=36)$, chymotrypsin $(0.3 \%, n=1)$ and chlorzoxazone $(4.2 \%$, $n=14)$. Caffeine was present in $1.5 \%(n=5)$ of the prescriptions. All of these drugs were part of pre-formulated commercially available drug formulations.

Drug dose was mentioned by $5.4 \%(n=18)$ dentists only. The peri-operative treatment protocol (pre-surgical dose followed by post surgical regimen) was preferred by $35.5 \%(n=118)$ dentists while $64.7 \%(n=215)$ gave the medication postoperatively only. The term 'Stat on Symptoms' was also mentioned by $21.6 \%(n=72)$ dentists. Post-operative prescription duration ranged from 0 to 9 days, the most common being 5 days.

The oral route was recommended by all 332 dentists with an additional injection via intramuscular route $(3.0 \%$, $n=10)$ or intravenous route $(1.5 \%, n=5)$ along with this.

Regarding steroids, out of the $33.7 \%(n=112)$ participants that prescribed steroids, dexamethasone $(50.8 \%, n=57)$ was the most preferred, followed by prednisolone $(29.4 \%$, $n=33$ ). Also, majority used generic names in their prescription $(72.3 \%, n=81)$ but only $19.6 \%(n=22)$ mentioned the dose. It is notable that most dentists preferred injectable steroid therapy (58.9\%, $n=66$ ) [Table 2].

\section{DISCUSSION}

A review of our study results shows that most of the dentists prescribed traditional NSAIDs to patients undergoing routine dental implant surgery. Only a marginal section of the dentists prescribed the newer cyclo-oxygenase selective group of drugs on a regular basis. Similar prescription patterns for analgesics in the dental setting have also been observed in studies conducted in Istanbul,,$^{[9]}$ Bangladesh, ${ }^{[10]}$ Karnataka $^{[11]}$ and Alabama. ${ }^{[12]}$ It is noteworthy that analogous results have also been reported in a study illustrating country comparisons in analgesic usage. ${ }^{[13]}$

Evaluation of the prescription patterns revealed that there was a great deal of variability in the drugs prescribed and the protocols followed for their prescription. Regarding the analgesic drug prescribed, ibuprofen was the most prescribed, followed by diclofenac. The role of both these drugs in the management of dental and post-surgical inflammatory pain is recommended by many authors. ${ }^{[14-17]}$ Amongst recommendations regarding the use of NSAIDs in dentistry, it has been suggested that ibuprofen is an ideal prototype for consideration in pain of dental origin. ${ }^{[2]}$

A noteworthy aspect of our study was the finding that the most common prescription pattern was the use of ibuprofen in combination with paracetamol, and diclofenac with paracetamol. No dentist prescribed paracetamol alone as an analgesic drug. Guidelines on sedation and management of dental pain ${ }^{[16]}$ state that it is irrational to combine two or more NSAIDs in therapy, however, paracetamol or opioid analgesics are suitable for combination with NSAIDs whenever a combination is required in severe pain only. ${ }^{[18-21]} A$ review of literature related to ibuprofen-paracetamol combination shows that the two drugs tend to provide efficacious pain control in the dental model when used together. ${ }^{[22]}$

Additionally, our study participants have also prescribed a variety of other NSAIDs such as piroxicam and ketorolac. However, it may be noted that these drugs have not been recommended for pain control in the dental pain model and are known to have more severe side effects. ${ }^{[2,4]}$ Some dentists have also mentioned the use of unrelated NSAIDs in their prescriptions, indicating a subjective preference in prescribing different drugs to different patients.

Another matter for concern regarding the use of NSAIDs for analgesia is the incidence of side effects, specifically gastrointestinal (GI) bleeding. ${ }^{[23]}$ The safest drug in this regard has been noted as Ibuprofen. ${ }^{[24]}$ However, this has also been related to the frequency and duration of overall NSAID therapy. This may not be significant in normal healthy individuals taking short-term therapy for oral surgical related pain. ${ }^{[2,4,25]}$ 
In relation to prescription patterns, there was some variability in the preference for perioperative regimens versus postoperative prescription only. The role of a preoperative or loading dose of analgesic medication before surgery has been recommended as a form of preemptive analgesia. ${ }^{[19,26]}$ In our study, only $35.5 \%$ ( $n=118$ ) of our participants who preferred the peri-operative regimen gave due consideration to this method of analgesic prescription.

The duration of prescription ranged from a self-medication preference or "stat-on-symptoms" approach to the medication being prescribed for 2 to 9 days post-surgically. Most authors recommend prescription duration of 4 to 6 days, and most of our study participants also fell into this range. ${ }^{[16,27]}$ All dentists in our study recommended the oral route of delivery, while only a few used an additional injection of NSAID drugs. Again, most authors recommend only oral NSAID therapy for dental and postoperative pain. ${ }^{[16,27]}$

When considering the method of prescription, it was seen that fewer dentists wrote the brand names of the drugs prescribed. The majority preferred to use generic names of commercially available drugs, including pre-formulated drug combinations.

To manage NSAIDs associated adverse effects on the GI tract, a proton pump inhibitor or $\mathrm{H} 2$ receptor antagonist is usually recommended for patients that are at high risk of ulcers. ${ }^{[10]}$ Only five of our study participants mentioned these drugs in their prescriptions. Our study also illustrated that only a minority prescribed semi-synthetic opioid analgesics (tramadol and dextropropoxyphene) and this trend was also in accordance with indications for use of NSAIDs for dental and post-surgical pain..$^{[2,16]}$

The role of glucocorticoids as adjuncts for pain and edema has received interest in recent time. ${ }^{[28]}$ Literature has shown that the short-term use of corticosteroids is safe and provides analgesia in acute postoperative pain in healthy adults. ${ }^{[29]}$ However, the optimal dose, mode and timing of administration remain unclear. ${ }^{[30]}$ One meta-analysis has provided reliable evidence that dexamethasone is an ideal drug for ameliorating acute postoperative pain..$^{[5]}$ Of the $30 \%$ of our study sample that did prescribe steroids, the majority did prescribe dexamethasone, but the duration, mode of delivery and protocol were variable. It is noteworthy that many of the dentists in our study prescribed injectable steroid therapy for up to seven days postoperatively.

It was interesting to note that pre-formulated commercially available drug combinations contained additional drugs such as serratiopeptidase and chlorzoxazone. The role of a muscle relaxant in dental implant surgery remains unclear.

Review of current literature reveals that almost all authors are in favour of the use of conventional NSAIDs, specifically ibuprofen, ibuprofen with paracetamol and diclofenac, for the management of dental and postoperative pain and inflammation. These drugs are also easily available over the counter without prescription. ${ }^{[9-11,13]}$ The role of steroids, especially dexamethasone, as adjuncts in postoperative pain management has also been reviewed. NSAIDs are also associated with side effects such as GI bleeding and allergic reactions, and it is critical that these drugs be prescribed cautiously. ${ }^{[19,24,25]}$

Our study illustrates that the current trend of prescription of analgesic and anti-inflammatory drugs for routine oral implant surgery among Indian dentists is in accordance with the guidelines for management of dental pain worldwide. However, evidence of prescription of drugs not primarily indicated for dental pain management and protocols not consistent with current recommendations, is noteworthy. It is essential that, to ensure safe delivery and avoidance of potential complications arising due to these drugs, dental practitioners should be more aware of the appropriate dose, dosage regimen and indications for specific analgesics. Additionally, further research is required to assess the outcomes, including adverse effects, related to the prescription of these drugs for oral implant surgery.

\section{REFERENCES}

I. Becker DE, Phero JC. Drug therapy in dental practice: nonopioid and opioid analgesics. Anesth Prog 2005;52:140-9.

2. Becker DE. Pain management: Part I: managing acute and postoperative dental pain. Anesth Prog 2010;57:67-79.

3. Singh G, Ramey DR, Morfeld D, Shi H, Hatoum HT, Fries JF. Gastrointestinal tract complications of nonsteroidal anti-inflammatory drug treatment in rheumatoid arthritis. A prospective observational cohort study. Arch Intern Med 1996; 156:1530-6.

4. Ong KS, Seymour RA. Maximizing the safety of non-steroidal anti-inflammatory drug use for postoperative dental pain: an evidence-based approach. Anesth Prog 2003;50:62-74.

5. Turan A, Sessler DI. Steroids to ameliorate postoperative pain. Anesthesiology 200I;15:457-9.

6. Brodala N. Flapless surgery and its effect on dental implant outcomes. Int J Oral Maxillofac Implants 2009;24: I I8-25.

7. Raffa RB. Pharmacology of oral combination analgesics: rational therapy for pain. J Clin Pharm Therap 2001;26:257-64.

8. Datta R, Grewal Y, Batth JS, Singh A. Current trend of antimicrobial prescription for oral implant surgery among dentists in India. J Maxillofac Oral Surg 2013;DOI: 10.1007/s I 2663-0I3-0567-7.

9. Șermet S, Akgün MA, Atamer-Șimșek S. Analgesic prescription pattern in the management of dental pain among dentists in İstanbul. Marmara Pharm J 20I2; I6:4I-7.

10. Mahadi HM, Jamiur RK, Binte WT, Sharmin CS. Study on the use pattern of NSAIDS in some general and specialized hospitals of Bangladesh. Int Res J Pharm 2012;3:152-5.

I I. Jayanthi MK, Suresha RN. A study of prescribing patterns of NSAIDS in dental OPD of a tertiary care teaching hospital. Asian J Med Clin Sci 2013;2:27-9.

12. Barasch A, Safford MM, McNeal SF, Robinson M, Grant VS, Gilbert GH. Patterns of post-operative pain medication prescribing after invasive dental procedures. Spec Care Dentist 20 I I;31:53-7.

13. Abbott FV, Fraser MI. Use and abuse of over-the-counter analgesic agents. J Psychiatry Neurosci 1998;23:13-34.

14. Scottish dental clinical effectiveness programme. Drug prescribing for dentistry: a clinical guidance. 2nd ed. 20II. p. 5I-63. Available from: http:// www.sdcep.org.uk/index.aspx?o=2334. [Last accessed on 2015 Feb 02].

15. Ong CK, Lirk P, Tan CH, Seymour RA. An evidence-based update on non-steroidal anti-inflammatory drugs. Clin Med Res 2007;5:19-34.

16. Pozzi A, Gallelli L. Pain management for dentists: the role of ibuprofen. AnnStomatol 201 I;2:3-24.

17. Bhaskar H, Kapoor P, Ragini. Comparison of transdermal diclofenac patch with oral diclofenac as an analgesic modality following multiple premolar extractions in orthodontic patients: a cross over efficacy trial. Contemp Clin Dent 2010;1:158-63. 
18. Ozkan BT, Durmus E, Kalayc A, Kurban S, Akca CN. The evaluation of safety and analgesic efficacy of paracetamol and ibuprofen followed by impacted third molar surgery. Eur J Gen Med 2010;7:310-6.

19. Haas DA. An update on analgesics for the management of acute postoperative dental pain. J Can Dent Assoc 2002;68:477-82.

20. Merry AF, Gibbs RD, Edwards J, Ting GS, Frampton C, Davies E and Anderson BJ. Combined acetaminophen and ibuprofen for pain relief after oral surgery in adults: a randomized controlled trial. Br J Anaesth 2010;104:80-8.

21. Gómez-Moreno G, Guardia J, Cutando A, Calvo-Guirado JL. Pharmacological interactions of anti-inflammatory-analgesics in odontology. Med Oral Patol Oral Cir Bucal 2009;14:81-9.

22. Rawal N, Macquaire V, Catalá E, Berti M, Costa R, Wietlisbach M. Tramadol/ paracetamol combination tablet for postoperative pain following ambulatory hand surgery: a double-blind, double-dummy, randomized, parallel-group trial. J Pain Res 201 1;4:104-10.

23. Mehlisch DR. The efficacy of combination analgesic therapy in relieving dental pain. J Am Dent Assoc 2002; 133:86I-7I.

24. Waring WS, Robinson OD, Stephen AF, Dow MA, Pettie JM. Does the patient history predict hepatotoxicity after acute paracetamol overdose?
QJM 2008;101:121-5.

25. Jackson $\mathrm{CH}, \mathrm{MacDonald} \mathrm{NC}$, and Cornett JW. Acetaminophen: a practical pharmacologic overview. Can Med Assoc J 1984;131:25-32.

26. Kissin I. Preemptive analgesia. Anesthesiology 2000;93:I I 38-43.

27. Hersh EV, Kane WT, O’Neil MG, Kenna GA, Katz NP, Golubic S, Moore PA. Prescribing recommendations for the treatment of acute pain in dentistry. Compend Contin Educ Dent 201 I;32:22,24-30.

28. Bodnar J. Corticosteroids and oral surgery. Anesth Prog 2001;48:130-2.

29. Dahners LE, Mullis BH. Effects of non-steroidal anti-inflammatory drugs on bone formation and soft tissue healing. J Am Acad Orthop Surg 2004; 12:139-43.

30. Salerno A, Hermann R. Efficacy and safety of steroid use for postoperative pain relief. J Bone Joint Surg Am 2006;88:1361-72.

How to cite this article: Datta R, Grewal Y, Batth JS, Singh A. A survey of analgesic and anti-inflammatory drug prescription for oral implant surgery. Plast Aesthet Res 2015;2:51-5.

Source of Support: Nil, Conflict of Interest: None declared.

Received: 25-09-2014; Accepted: 18-12-2014 